EGU2020-19991

https://doi.org/10.5194/egusphere-egu2020-19991

EGU General Assembly 2020

(c) Author(s) 2021. This work is distributed under

the Creative Commons Attribution 4.0 License.

\title{
End-Member Modeling Analyses (EMMA) of pseudo-Thellier style experiments to derive absolute paleointensities from lavas
}

\author{
Liz van Grinsven, Tristan van Leeuwen, and Lennart de Groot \\ Utrecht University, Earth Sciences, Netherlands (liz_van_grinsven@live.nl)
}

Over the past years several groups have made efforts to calibrate the 'pseudo-Thellier' technique to obtain paleointensities from materials that acquired their natural remanent magnetizations thermally, while avoiding heating the samples during the experiments. These calibrations revolve around mapping laboratory induced Anhysteretic Remanent Magnetizations (ARMs) to thermally acquired Natural Remanent Magnetizations (NRMs).

One approach has been to plot pseudo-Thellier slopes against paleointensities that are either known (for very young lavas) or result from different paleointensity techniques. Although the obtained calibration relation is linear and closely follows the data, the relation worryingly misses the origin, i.e. a pseudo-Thellier slope of 0 leads to a paleointensity of up to $14.7 \mu \mathrm{T}$. Currently, there is no satisfying explanation for this non-zero axis intercept. Another approach has been to calibrate the mapping between the TRM and ARM by giving (thermally stable) samples a remanent magnetization and force the calibration through the origin. Although to the current state of our knowledge this is theoretically correct, the mismatch between the calibration relation and the data introduced by this approach is evident. So far, neither of these approaches yielded a generically applicable and theoretically acceptable mapping between ARMs and TRMs.

Naturally occurring basalts, however, are assemblages of magnetic minerals differing in grain size, shape, and chemistry. Here we take a new approach to the interpretation of pseudo-Thellier data by trying to find end-members for the ARMs, through nonnegative matrix factorization, that represent these different magnetic minerals in the samples. With the idea that the quantity of these end-members in the different ARMs are related to the original NRMs intensities. We use a set of 580 samples from different volcanic edifices (Hawaii, Mt. Etna, Tenerife, Gran Canaria, and Iceland) that recently cooled in the Earth's magnetic field, so in known field strengths. The first results that we will present are encouraging and address the current challenges with obtaining absolute paleointensities from lavas with a pseudo-Thellier approach. 